\title{
FGF signalling is involved in cumulus migration in the common house spider Parasteatoda tepidariorum
}

Ruixun Wang ${ }^{1}$, Linda Karadas ${ }^{1}$, Philipp Schiffer ${ }^{1}$, Matthias Pechmann ${ }^{1 *}$

1 Institute for Zoology/Developmental Biology, Biocenter, University of Cologne, Zuelpicher Str. 47b, 50674 Cologne, Germany

*Corresponding author

\begin{abstract}
Cell migration is a fundamental component during the development of most multicellular organisms. In spiders, the collective migration of a signalling centre, known as the cumulus, is required to set the dorsoventral body axis of the embryo.

Here, we show that FGF signalling plays an important role during cumulus migration in the spider Parasteatoda tepidariorum. Spider embryos with reduced FGF signalling lack cumulus migration and display dorsoventral patterning defects. Our study reveals that cumulus expression of several FGF signalling components is regulated by the transcription factor Ets4. In conjunction with a previous study, we show that the expression of $f g f 8$ in the germ-disc is regulated via the Hedgehog signalling pathway. We also demonstrate that FGF signalling influences the BMP signalling pathway activity in the region around cumulus cells.

Finally, we show that FGF signalling might also influence cumulus migration in basally branching spiders and we propose a hypothetical model in which $f g f 8$ acts a chemoattractant to guide cumulus cells towards the future dorsal pole of the spider embryo.
\end{abstract}

\section{Keywords}

Cell migration, spider, cumulus, DV axis, FGF, Hedgehog, BMP, Ets4

\section{Introduction}

In vertebrates like mice and humans, almost two dozen of ligands and four receptors are responsible for the regulation of one of the most complex signalling pathways, the Fibroblast-Growth-Factor-Receptor (FGFR) signalling pathway. Differential splicing of the receptors and different ligand/receptor combinations are able to regulate a wealth of functions including axis establishment, cell proliferation, differentiation and survival (reviewed in $[1,2]$ ). In contrast, the genomes of invertebrates such as beetles or flies 
only have a handful of fibroblast growth factor receptors and ligands ([3-5] and reviewed in [6]). Nevertheless, also in these animals FGFR signalling is involved in a variety of important developmental processes, which include the formation of the mesoderm or the branching of the tracheal system (summarized in [4,6]). In Drosophila, these two processes involve the regulation of directed cell migration, another widely conserved feature of the FGFR-signalling pathway. By acting as chemo-attractants, FGFs are able to attract cells by altering components of the cytoskeleton, which in turn leads to the formation of filo- and lammellopodia (e.g. [7,8], reviewed in [6]).

In the nematode Caenorhabditis elegans, EGL-15 and EGL-17 (the FGF receptor and FGF orthologs of $C$. elegans) are involved in a chemo-attractive mechanism to guide sex myoblasts to their final destination [9-11].

Also in vertebrates FGF signalling controls cell migration in various tissues. In mouse embryos, endoderm- and mesoderm-derived tissues depend on the outward migration of cells from the primitive streak, and this process is controlled by a small subset of FGFs [12].

Setting up the dorsoventral (DV) body axis of chelicerate species, like spiders, involves the migration of the cumulus (a cluster of cells that possesses organizing capacities). Cumulus cells are inducing the DV body axis via the secretion of Dpp (the BMP2/4 ortholog) and the subsequent activation of the BMP signalling pathway in a subset of germ-disc cells [13-19]. As the name implies, the germ-disc is radially symmetric and gives rise to the embryo proper. Cumulus transplantation or the local activation of the BMP signalling pathway demonstrated that all germ-disc cells have the capacity to develop either into ectodermal embryonic or into dorsal field cells [18,20,21]. For this reason, the decision if a germ-disc cell develops into ectoderm or changes its fate towards a dorsal field cell depends on the signalling and the direction of cumulus migration.

It is still unclear if the direction of cumulus migration in the radially symmetric field of germ-disc cells is actively guided via unknown factors, or if cumulus migration is a completely stochastic process. Hedgehog signalling was found to play a role in cumulus migration $[22,23]$. However, it is unknown if Hedgehog is directly or indirectly involved during this important developmental process.

Here we show that cumulus migration in the common-house spider Parasteatoda tepidariorum is highly dependent on FGF signalling. We show that a FGF ligand is asymmetrically expressed in the early germ-disc of many spider embryos and we 
propose a hypothetical model in which FGF signalling is involved in the guidance of the cumulus cells towards the periphery of the germ-disc.

\section{Methods}

\section{Spider husbandry and embryology}

For all experiments we used our Cologne laboratory culture of Parasteatoda tepidariorum. Adults and juveniles of Parasteatoda tepidariorum were kept in plastic vials at room temperature and were fed with Drosophila melanogaster and crickets (Gryllus bimaculatus). Embryos were collected and fixed as described previously [19] and staged according to [24]. Acanthoscurria geniculata embryos were kept as described previously [18]. Adults and juveniles of Steatoda grossa were collected near Cologne (Hürth, Germany) and were kept under the same conditions as Parasteatoda tepidariorum.

\section{Gene cloning}

CLC Main Workbench 7 (QIAGEN Aarhus A/S) was used to perform a local tBLASTn [25] against the Parasteatoda tepidariorum official AUGUSTUS gene set (https://i5k.nal.usda.gov/Parasteatoda_tepidariorum, [26]). For this, protein sequences of known homology were downloaded from FlyBase [27] and NCBI. Putatively identified genes were reciprocally BLASTed against the online NCBI nr database using BLASTx to confirm their identity.

PCR amplification and cloning of genes was performed using standard techniques. Genes were cloned into the pCR4 and the pCRII vector (ThermoFisher scientific). Pt-Ets4, Ptfork-head, Pt-orthodenticle, Pt-short-gastrulation, Pt-decapentaplegic, Pt-hedgehog and Pt-caudal were isolated previously [19,28,29].

A Pt-fgf8 DNA fragment of approximately $2 \mathrm{~kb}$, including the full coding sequence and a portion of the 5'UTR and the 3'UTR, was amplified using Pt-fgf8-Fw (5'CATCTCTTCGCTCTCCGCGC-3') and Pt-fgf8-Rev (5'-GAATGCTCGTGCAAAGAGAGTG-3').

Pt-dof, Pt-fgf1, Pt-FGFR1 and Pt-FGFR2 were amplified using Pt-dof-Fw (5'GAAATGGCTCCTGTCGACGTTAC-3'), Pt-dof-Rev (5'-CAATACTGGAACAGGTTGAGCTG-3'), Pt-fgf1-Fw (5'-GTGGatAGAGGCATACCGAGT-3'), (5'CGGAACACCTCTACGGAACG-3'), Pt-FGFR1-Fw (5'-GACATATGCTGAGGAAGATAATAG-3'), 
GTCACAGTCATTTTAGGCTTG-3') and Pt-FGFR2-Rev (5'-CAGTGTACATCTCCTGAGGTAC3') primer, respectively.

The published transcriptome [18] was searched for Acanthoscurria geniculata fgf1, fgf8 and $f g f 17$ sequences. Total RNA was isolated and cDNA was produced as described in [18]. Ag-fgf gene sequences were isolated using the following primer combinations: Agfgf1-Fw (5'-GTG CAA CGA GGA CAA CTA TTC AG-3') and Ag-fgf1-Rev (5'-GAG TAC TAT ACA TTT CAA AAC ACC G-3'); Ag-fgf8-Fw (5'-CTC CAT GCT TCA CCG TTG ATG-3') and Ag-fgf8-Rev (5'-CTG CTG TCA TTG GCA CTT GTC-3'); Ag-fgf17-Fw (5'-GAA CAG CCT GAG CAA ATG CCA C-3') and Ag-fgf17-Rev (5'-CAC CTG AGA ATC TTG CTG GAC AC-3').

Nucleotide sequences of $f g f 8$ and $f g f 17$ of different spider species were blasted against the combined RNA-Seq from 19 Libraries sequenced from False Black Widow (Steatoda grossa) (SRR1539523). Blast hits were manually aligned and primer sequences of Steatoda grossa $f g f 8$ and $f g f 17$ were designed according to this alignment. Total RNA of Steatoda grossa was extracted using TRIzol Reagent (life technologies) and cDNA was produced using the RNA to cDNA EcoDry Premix (TaKaRa Clontec, double primed). $S g$ fgf8 and $S g$-fgf17 were amplified using Sg-fgf8-Fw (5'-CAG GTT ACT ACA GAT TGC CTC C-3') and Sg-fgf8-Rev (5'-GTA CGT GTG CTC CCT CTT GAT G-3') and Sg-fgf17-Fw (5'-GTT CAT CTG CAC TTC TGT CAT GG-3') and Sg-fgf17-Rev (5'-CCG TGT GTC ATT CCA GAG ATG-3') primer, respective.

\section{Transcriptome sequencing of cumulus cells}

To knock down Pt-Ets4, several spider females were injected with Pt-Ets4 dsRNA (nucleotide 11-697 of aug3.g4238.t1 was used for the knockdown) as described in [19]. While the majority of stage 4 control (dsRed dsRNA was used for the control injections) and Pt-Ets4 RNAi embryos of $3^{\text {rd }}$ and $4^{\text {th }}$ cocoons were formaldehyde fixed and stored in $100 \% \mathrm{MeOH}$ at $-20^{\circ} \mathrm{C}$, around 50 embryos of each cocoon were monitored under oil. Subsequently, the primary thickening of fixed control embryos and of fixed embryos where the siblings of the same cocoon showed the characteristic Pt-Ets 4 knockdown phenotype (see [19]) was extracted using sharp needles. The primary thickening of 100120 embryos was extracted for each replicate. For each condition (control vs. Ets4 RNAi) the embryos of three independently produced cocoons were used. Overall, three biological and technical replicates were produced per condition. The total RNA of the extracted cells was isolated using the Quick-RNA ${ }^{\mathrm{TM}}$ FFPE Kit (Zymo Research). Library preparation and sequencing was carried out at the Cologne Center for Genomics, using a 
paired-end 2x150nt approach on the Illimuna NovaSeq platform. The sequenced reads were subjected to adaptor and quality threshold trimming using fastp [30]. Cleaned reads where mapped against the published $P$. tepidariorum gene set [26] using kallisto [31] and the expression patterns analysed in Degust (David Powell (2015), drpowell/degust v3.2.0 (v3.2.0); Zenodo; https://doi.org/10.5281/zenodo.3258933).

\section{Phylogenetic analysis}

The FGF core sequence (see [3]) was used to align FGF proteins of different species. In case of Dof related proteins, only the conserved DBB (Dof, BCAP, BANK region) motif as well as the region of the ankyrin repeats were used to generate the phylogeny. Alignments were produced using MUSCLE [32] and aligned sequences were trimmed using TrimAl with the GappyOut setting [33]. Maximum likelihood based phylogenies were constructed using PhyML at 'phylogeny.fr' [34]. Final phylogenies were generated with the WAG substitution model and 1000 bootstrap replicates [35]. Gene bank accession numbers or sequences can be found in the in supplemental information (figure legends of Fig. S1 and S5 and Supplemental Sequences).

\section{Parental RNAi experiments}

Adult spider females were injected 3-4 times (in a 2-3 day interval) with $2 \mu \mathrm{l}[2-3 \mu \mathrm{g} / \mu \mathrm{l}]$ dsRNA solution. Pt-ptc and Pt-Ets4 pRNAi experiments were performed as described in [19]. Double stranded RNA was produced using the T7 MEGAscript Kit (ThermoFisher scientific).

During the initial RNAi screen Pt-fgf8 (AUGUSTUS prediction: aug3.g5611.t1; Schwager et al. 2017) was amplified using the g5611-Fw (5'-CAGGTTACTACAGATTGCCTCC-3') and g5611-Rev (5'-GCACTTTCGTTCGTATTCATAG-3') primer. Template to generate dsRNA was amplified using the T7 overhang primer T7-g5611-Fw (5'GTAATACGACTCACTATAGGGCTAGCGTACCTGTGT-3') and T7-g5611-Rev (5'GTAATACGACTCACTATAGGGGCCAGTCCCCAGC-3'). To test for "off-target-effects", two non-overlapping DNA fragments coding for Pt-fgf8 were amplified using T7-Pt-fgf8-off1Fw (5'-GTAATACGACTCACTATAGGGCTCCGCGCTGCGGC-3') and T7-Pt-fgf8-off1-Rev (5'GTAATACGACTCACTATAGGGCGCCTCAATAGTGGAGC-3') and T7-Pt-fgf8-off2-Fw (5'GTAATACGACTCACTATAGGGGTGTGTCTATTCAAAGAAGG-3') and T7-Pt-fgf8-off2-Rev (5'-GTAATACGACTCACTATAGGGGGATGATGAGAGATCTATAG -3'), respectively. These DNA fragments were used as a template to generate dsRNA that targeted independent 
regions of the Pt-fgf8 transcript. For the statistics shown in Fig. S2, four spider females were injected with dsRNA of each "off-target" fragment.

The statistics shown in Fig. S5 1 (knockdown of Pt-dof) is based on three injected spider females.

Pt-hh was cloned into pCRII vector using Pt-hh-Fw (5'GGTACACCCATAAATGCCGTCAGTTGAG-3') and Pt-hh-Rev (5'GTATATTCATGACAAGCGCCAGATCACACC-3'). Subsequently, T7 and T7M13R primer were used to generate the template for dsRNA production. Several spiders were injected with the generated Pt-hh dsRNA and only severely affected cocoons were used for the experiments shown in this study.

\section{Embryo fixation, in situ hybridisation and pMad antibody staining}

To analyse BMP pathway activity in control and Pt-fgf8 knockdown embryos a pMad antibody staining was performed as described in [19]. Fluorescent pMad antibody staining was performed using the Phospho-Smad1/5 (Ser463/465) (41D10) Rabbit mAb (Cell Signaling Technology, Inc.; antibody concentration: 1:1000) as primary and an Alexa488 anti rabbit AB (Invitrogen; 1:400 concentration) as secondary antibody.

\section{In situ hybridisation}

In situ hybridisation was performed as described previously $[19,36]$. Fluorescent in situ hybridisation was performed using FastRed [37]. Double in situ hybridisation was performed using NBT/BCIP and INT/BCIP as a substrate.

\section{Imaging and image analysis}

Embryos were imaged using an Axio Zoom.V16 that was equipped with an AxioCam 506 colour camera. Confocal imaging was performed on a LSM 700 (Zeiss). Projections of image stacks were carried out using Helicon Focus (HeliconSoft) or Fiji [38]. Live imaging was carried out on the Axio Zoom.V16 and images were processed using Fiji. Living embryos were monitored under Voltalef H10S or Halocarbon 700 (Merck) oil and live imaging was performed at RT.

Movies were created using Fiji. Images have been adjusted for brightness and contrast using Adobe Photoshop CS5.1.

False-colour overlays of in situ hybridization images were generated as described in [19]. 


\section{Results}

\section{Cumulus migration requires FGF signalling}

A RNAi screen, designed to find new genes that are involved in the process of axis specification in P. tepidariorum (see [19]) revealed that also FGF signalling might have an important role during axis formation in spiders.

During this RNAi screen, the knockdown of a gene, orthologous to $f g f 8$ (named Pt-fgf8 in this study; AUGUSTUS genome prediction: aug3.g5611.t1; see Fig. S1), led to a strong dorsoventral axis patterning defect phenotype in a portion of the analysed embryos. Time lapse imaging of $P t-f g f 8$ knockdown embryos revealed that the down regulation of Pt-fgf8 is preventing cumulus migration at stage 5 of development (Fig. 1 a, b; Movie S1). In control embryos, morphogenetic processes lead to the opening of the germ-disc and to the formation of the bilaterally symmetric germ-band embryo owing a clear dorsoventral polarity (Fig. 1 a, b; Movie S1). This was in stark contrast to Pt-fgf8 pRNAi embryos in which germ-disc opening did not occur. As a result, the knockdown embryos had a tube-like morphology at stages 7-10 (Fig. 1 a, b; Movie S1). As revealed by an in situ hybridisation for the ventral cell fate marker short gastrulation (Pt-sog) and the anterior marker orthodenticle (Pt-otd), stage 8 Pt-fgf8 knockdown embryos were completely ventralized and were lacking any bilateral symmetry (Fig. $1 \mathrm{c}-\mathrm{g}$ ). However, the staining of Pt-otd at the anterior and caudal (Pt-cad) at the posterior pole indicated that anterior and posterior patterning was not affected by the knockdown of Pt-fgf8 (Fig. $1 \mathrm{~g}, \mathrm{~g}^{\prime}$ and i).

As already mentioned, only a portion of the Pt-fgf8 RNAi embryos showed a DV patterning phenotype in the initial knockdown experiment. In this initial RNAi experiment almost the complete coding sequence of $P t-f g f 8$ was used to generate dsRNA for parental injection. To verify and quantify this observation and to rule out that this low knockdown efficiency was fragment specific, we tested two non-overlapping

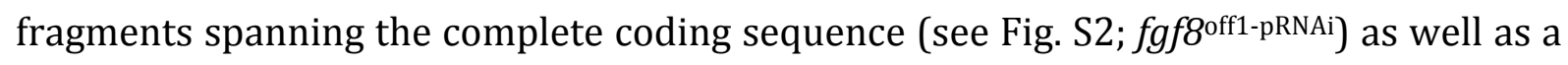
large region of the 3'UTR of Pt-fgf8 (see Fig. S2; fgf8 off2-pRNAi). For both dsRNA fragments we observed a very similar number of embryos showing the described cumulus migration and DV defect phenotype (Fig. S2). The highest number of affected embryos was present in the third cocoon (around 3 weeks after injection, see Fig. S2). However, similar to the initial approach, only around $25 \%$ of the embryos were affected after the knockdown of $P t-f g f 8$. To overcome the low knockdown efficiency we were searching for 
other components of the FGF signalling pathway. Scanning the Parasteatoda tepidariorum genome [26], we found two paralogous genes coding for FGF receptors (named Pt-FGFR1 (aug3.g26565.t1) and Pt-FGFR2 (aug3.g26271.t1) in this study) and an ortholog of a gene known as downstream-of-fgf (dof), stumps (sms) or heartbroken (hbr) (named Pt-dof in this study; aug3.g4286.t1). We analysed the expression of the genes and found that Pt-FGFR1 and Pt-dof were expressed in the migrating cumulus of stage 5 embryos, while Pt-FGFR2 did not show any expression at germ-disc stage (Fig. S3-S5). As expected from a previous analysis [22] the knockdown of the two $f g f$ receptors did not lead to any detectable defect in cumulus migration. In contrast, some of the Pt-dof RNAi embryos showed a phenotype that was identical to the knockdown of Pt-fgf8 (Fig. S5i). However, as the Pt-dof knockdown did not result in a higher frequency of strong FGF signalling dependent cumulus migration defect phenotypes (Fig. S5l), we used the $P t-f g f 8$ RNAi embryos for the further analysis.

In conclusion, the knockdown of Pt-fgf8 as well as of the downstream effector Pt-dof prevents cumulus migration in the spider Parasteatoda tepidariorum. As cumulus migration is required for the formation of the dorsoventral body axis, FGF signalling is an important player during early spider embryogenesis. 

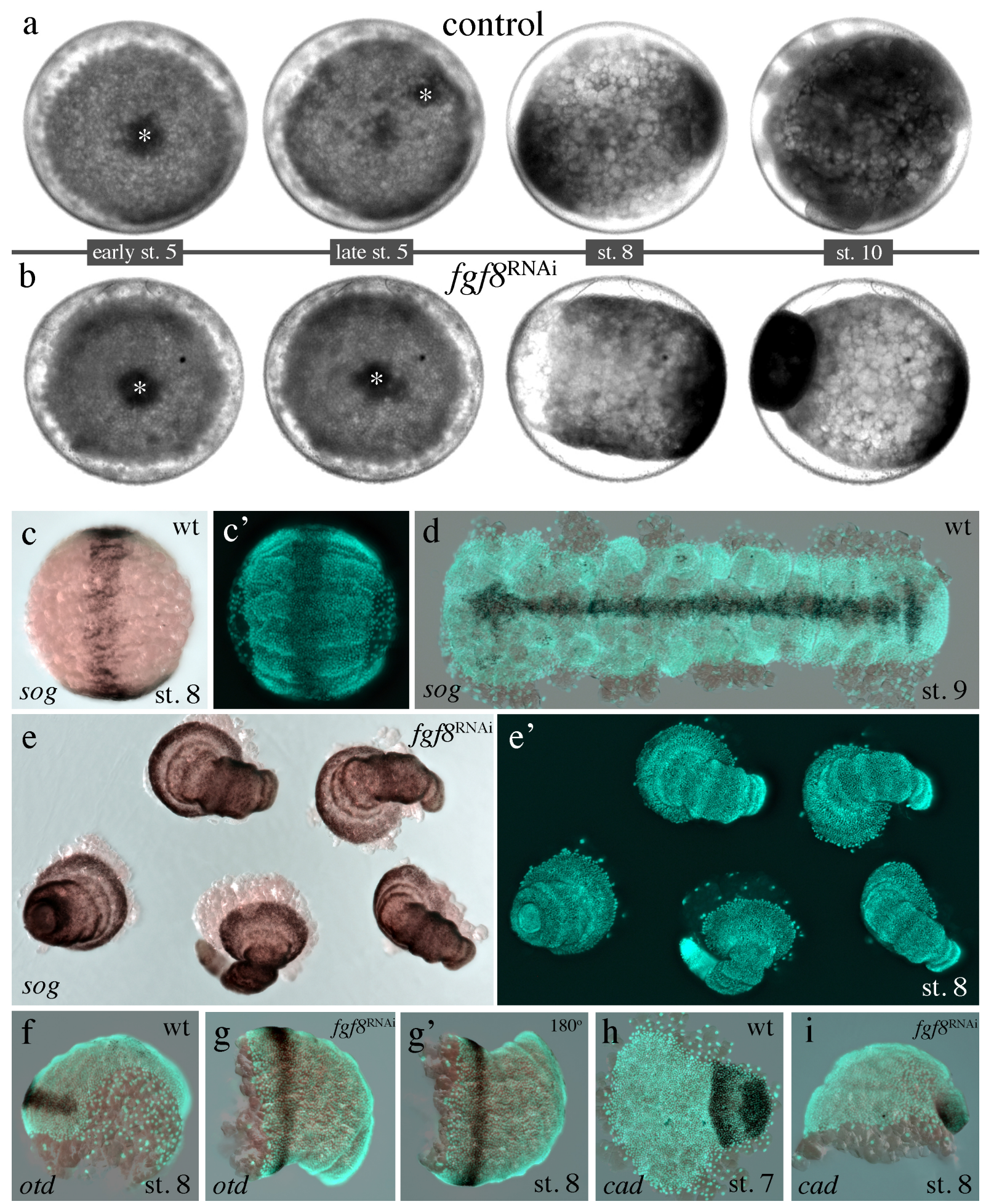

Fig. 1 Knockdown of $\boldsymbol{P t}$ - $f g f 8$ prevents cumulus migration. (a, b) Stills from Movie S1. In contrast to the control embryo, the cumulus (marked by the asterisk in $\mathbf{a}$ and $\mathbf{b}$ ) of $P t$ fgfo RNAi embryo is not shifting and stays in the centre of the germ-disc during stage 5 of embryonic development. Pt-fgfo knockdown embryos are radially symmetric, ventralized and have a tube-like morphology during later stages of development $(\mathbf{b}, \mathbf{e}, \mathbf{g}$, i). (c-i) Expression of a ventral (Pt-sog), an anterior (Pt-otd) and a posterior (Pt-cad) marker gene in control and Pt-fgf8 RNAi embryos. Whole mounted (c, e, f, g, i) and flat mounted $(\mathbf{d}, \mathbf{h})$ embryos co-stained with the nuclear dye Sytox green. To clearly show the radially symmetry, the embryo shown in g was turned by $180^{\circ}\left(\mathrm{g}^{\prime}\right)$. 


\section{Dynamic germ-disc expression of Pt-fgf8}

It is known that FGF ligands can function as a chemo attractant to attract cells that express a FGF receptor (reviewed in e.g. [6]). For this reason, we were very interested to see whether Pt-fgf8 might be expressed in a subset of germ-disc cells to be able to guide the Pt-FGFR1 expressing cumulus cells towards the rim of the germ-disc.

At stage 3 and 4, Pt-fgf8 transcripts were detectable within the central primary thickening of the germ-disc (Fig. 2 a-c). Confocal sectioning of fluorescently labelled stage 4 embryos revealed that Pt-fgf8 transcripts were present in the gastrulating cells that entered the germ-disc via the blastoporus (see Fig. 2 c).

Single cells or groups of ectodermal germ-disc cells started to express Pt-fgf8 (Fig. 2 b', Fig. 2 c) at the beginning of stage 4 . At the end of stage 4 (Fig. 2 d-e, Fig. S6) many embryos showed a clear asymmetric Pt-fgfo expression. In extreme cases, half of the germ-disc was positive while the other half was mostly negative for Pt-fgf8 transcripts (Fig. 2 e, Fig. S6 a, c, e, f, i). However, at this stage every embryo showed a unique pattern and the expression of $P t-f g f 8$ was very dynamic and variable. While in some embryos $P t$ fgf8 could be detectable in only a few germ-disc cells (e.g. Fig. S6 b, g) other embryos showed a nearly ubiquitous expression of Pt-fgf8 (e.g. Fig. S6 d, h). At late stage 4, expression seemed to be switched off from the primary thickening and was restricted to the ectodermal cells of the germ-disc (e.g. Fig. 2 d; e.g. Fig. S6 b, g ,j).

A similar dynamic and variable expression was also detectable at early and mid stage 5 embryos when the cumulus cells started to shift towards the rim of the germ-disc. At these stages, some embryos showed little (Fig. S7 c, e, g, h) or a patchy, salt and pepper like expression of Pt-fgf8 (Fig. 2 f, h e.g. Fig. S7 i), others had a ring-like expression domain (Fig. 2g, Fig. S7 j-m) and single embryos showed no Pt-fgf8 expression (Fig. S7 f). Importantly, during stage 5, Pt-fgf8 expression was always switched of from the centre of the germ-disc (Fig. 2 f-j, Fig. S7-S9).

In contrast to this dynamic and variable expression until mid stage 5 , we could observe a very consistent feature of Pt-fgf8 expression at late stage 5. In all analysed embryos $(n>30)$ the cumulus did reach the rim of the germ-disc at high levels of Pt-fgf8 expression (Fig. 2 i-k, Fig. S8 and S9). Confocal sectioning of fluorescently labelled embryos revealed that Pt-fgf8 transcripts were detectable in ectodermal, pMad positive, germ-disc cells (Fig. 2 k). For several embryos, we even had the impression, that ectodermal, $P t-f g f 8$ expressing cells were directly anterior to the travelling cumulus cells (e.g. Fig. 2i). 
Overall, Pt-fgf8 showed a very dynamic expression during germ-disc stages and every embryo had its unique pattern. However, the very asymmetric expression at late stage 4 and the strong expression of Pt-fgf8 at the final position of the cumulus at late stage 5 suggested that Pt-fgf8 might function as a chemo attractant to guide the cumulus cells towards the rim of the germ-disc.
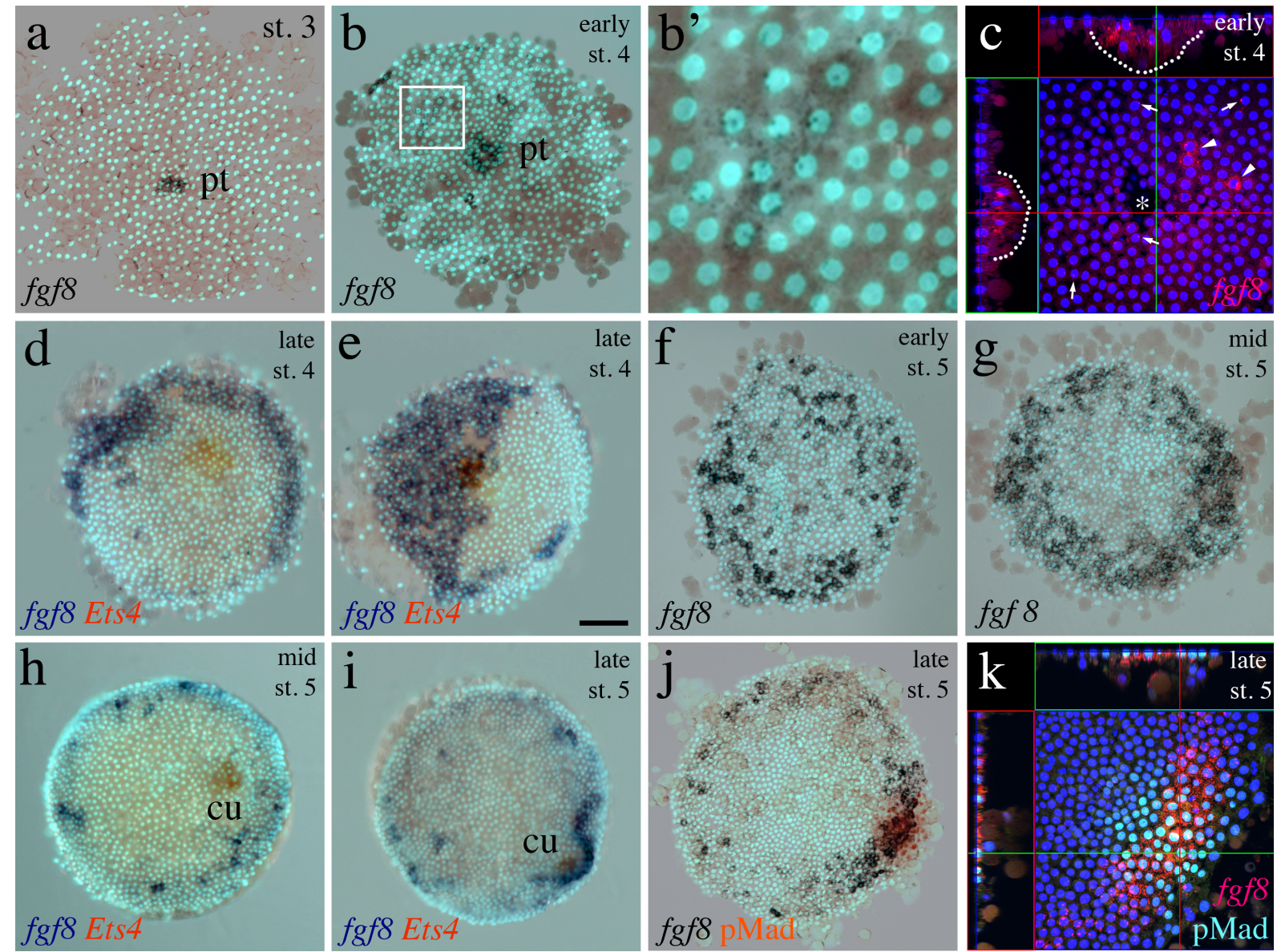

Fig. 2 Dynamic expression of Pt-fgf8 in the germ-disc. (a-c) At stage 3 and 4, cells delaminate via the blastopore (see asterisk in c) and form the primary thickening (pt in a and b). The cells of the primary thickening are positive for Pt-fgf-8 transcripts (outlined by the dotted line in the orthogonal views in c). At early stage 4, Pt-fgfo is stochastically activated in single or in groups of cells within the ectoderm of the germdisc (see boxed region in $\mathbf{b}$ and arrows and arrow heads in $\mathbf{c}$ ). Nascent transcripts in the nuclei (arrow heads) and cells with Pt-fgf8 transcripts in the cytoplasm (arrows) of the germ-disc cells are indicated in c. Boxed region in b is magnified in b'. (d-g) At late stage 4 and early stage 5 , embryos show broad, asymmetric (d, e), spotted (f) or ring like (g) $P t$-fgf8 expression within the germ-disc. At mid and late stage 5, most embryos show strong expression of $P t-f g f 8$ in the region around the migrating cumulus (cu). ( $\mathbf{j}$ and $\mathbf{k})$ Ectodermal expression of $P t-f g f 8$ in the germ-disc cells overlaying the cumulus. Double in situ hybridisation showing Pt-fgf8 expression in blue and the cumulus marker Pt-Ets4 in orange ( $\mathbf{d}, \mathbf{e}, \mathbf{h}-\mathbf{j})$. $\mathbf{c}$ and $\mathbf{k}$ show confocal scans of fluorescently labelled embryos (via pMad antibody staining and in situ hybridisation using fast red as a substrate). All embryos were co-stained with the nuclear dye DAPI or Sytox green. Pt-fgf8 expression beyond stage 5 is depicted in Fig. S10. 


\section{Possible ancestral function of FGFR-signalling in cumulus migration}

Searching for fibroblast growth factor like proteins revealed that the P. tepidariorum genome possesses two genes that code for FGFR ligands. This is in contrast to many other spider species where we could find three FGFs. Phylogenetic analysis revealed that the spider FGFs fall into the FGF A and D families (FGF families according to [39], see Fig. S1). All analysed spider species have a clear homolog for Fgf1 and Fgf8. Only P. tepidariorum is missing a homolog for Fgf17, which is present in other spider transcriptomes/genomes. As the genome of $P$. tepidariorum is well assembled and several transcriptomic resources are available for this species $[26,40]$, it is very likely, that $f g f 17$ was lost in the lineage leading to Parasteatoda.

To see whether Fgf17 might have a role during cumulus migration in other spider species, we cloned $f g f 17$ and analysed its expression in Steatoda grossa (S. grossa, a cobweb spider species, closely related to P. tepidariorum) and Acanthoscurria geniculata (a basally branching mygalomorph spider species). Live imaging of $S$. grossa embryos revealed, that embryogenesis is very similar to P. tepidariorum embryogenesis (see Movie S2). Expression analysis revealed that S. grossa $f g f 17$ ( $S g-f g f 17)$ seems not to be expressed at germ-disc stages (Fig. S11 b). This is in contrast to $S g$-fgf8 that shows a similar and variable expression (see Fig. S11a), as was shown for Pt-fgf8 in germ-disc stage P. tepidariorum embryos (e.g. Fig. 2). A. geniculata fgf8 (Ag-fgf8) and fgf17 (Agfgf17) showed both a variable (sometimes enhanced expression in the region of the cumulus) expression pattern at stage 5 of embryogenesis (Fig. S12 g and h). Like in P. tepidariorum, also Ag-FGFR1 (but not $A g$-FGFR2) and Ag-dof showed an expression in cells of the migrating cumulus (Fig. S12 c-e). This analysis indicated that also in basally branching spiders FGF signalling might be involved in cumulus migration. For $f g f 1$ we could not detect any specific embryonic localisation of transcripts, neither in $P$. tepidariorum nor in A. geniculata (Fig. S12 f, Fig. S13 a, Fig. S14).

\section{Cumulus expression of FGF signalling components are regulated by the transcription factor Pt-Ets 4}

The primary thickening of stage 4 embryos is a cluster of gastrulating cells that enter the germ-disc via the blastoporus [13-15,41]. In P. tepidariorum, around nine cells of the primary thickening will contribute to the cell cluster of the migrating cumulus [13]. In 
2017, we showed that the transcription factor Pt-Ets4 is expressed in the cells of the primary thickening (see Fig. 3a) and in the migrating cumulus [19]. We identified PtEts4 as an important factor for maintaining the cohesion of the cumulus cells and could show that Pt-Ets4 is required to activate cumulus specific genes. Furthermore, ectopic expression of Pt-Ets4 was able to induce cell delamination and migration [19].

As described above, Pt-fgf8 (Fig. 2 a-c), Pt-FGFR1 (Fig. S3 a) and Pt-dof (Fig. S5 b) were expressed in the region of the primary thickening and were likely to be co-expressed with Pt-Ets4. To see whether Pt-Ets4 might be involved in the transcriptional activation of the FGF signalling components we analysed the expression of Pt-FGFR1 and Pt-dof in stage 4 Pt-Ets 4 RNAi embryos. Indeed, while the expression of Pt-fkh, a gene known being expressed in the primary thickening but not being regulated by Pt-Ets4, was unchanged, Pt-FGFR1 expression was completely absent in Pt-Ets4 RNAi embryos (Fig. 3 c, f). Also the expression of Pt-dof was greatly reduced upon Pt-Ets4 knockdown (Fig. S5 j, k). In addition, a differential expression analysis of genes being regulated by Pt-Ets4 at stage 4 confirmed that Pt-FGFR1, Pt-dof, and Pt-fgf8 have a significantly reduced expression profile (see table 1 ).

Table1: Expression changes (TPM values) of FGF signalling components after RNAi with Pt-Ets4.

\begin{tabular}{|l|l|l|l|l|l|}
\hline gene & FDR & dsRed & dsRed & Ets4 RNAi & Ets4 RNAi \\
\hline Ets4 & $2,55 \mathrm{E}-31$ & 161,599 & 196,816 & 2,20942 & 3,25887 \\
\hline fgf8 & $4,87 \mathrm{E}-18$ & 46,3583 & 80,273 & 6,65353 & 4,35487 \\
\hline dof & $1,54 \mathrm{E}-20$ & 9,30084 & 6,15884 & 0,238895 & 0,094327 \\
\hline fgfr1 & $1,86 \mathrm{E}-13$ & 9,56246 & 8,5299 & 0,82618 & 0,580557 \\
\hline fgfr2 & 0,64875295 & 2,48446 & 2,67093 & 2,5176 & 1,55443 \\
\hline
\end{tabular}

\section{Knockdown of $P t-f g f 8$ is influencing the activation of the BMP signalling pathway}

As shown in previous studies, the down regulation of the Hedgehog receptor Patched (Pt-ptc) is also able to inhibit cumulus migration $[19,22]$. However, there is a fundamental difference between the Pt-ptc and the Pt-fgf8 knockdown phenotype.

In wild type embryos, the shifting and Dpp secreting cumulus leads to the activation of the BMP signalling pathway at the periphery of the germ-disc. This is leading to the formation of the dorsal field, which is required to transform the radially symmetric germ-disc into a bilaterally symmetric germ-band. The cumulus cells of the Pt-ptc RNAi embryos are still able to activate the BMP signalling pathway in nearby ectodermal germ-disc cells [22]. However, as the cumulus of Pt-ptc knockdown embryos was not 
shifting, the dorsal field was established in the centre of the germ-disc. An ectopic formation of the dorsal field in the centre of the germ-disc did never occur in Pt-fgf8 RNAi embryos (Fig. 1 and Movie S1). This suggested to us that FGFR signalling is required to activate the BMP signalling pathway in stage 5 spider embryos.

To see at what level the FGFR signalling pathway was required to activate the BMP signalling pathway, we performed an in situ hybridisation to detect Pt-dpp transcripts and performed a pMad antibody staining to analyse BMP pathway activity (Fig. 3 g-j). For this experiment, we took 338 embryos of a third cocoon (produced by a spider female that was injected with Pt-fgf8 dsRNA) and divided the embryos in four groups. We took advantage of the fact that $P$. tepidariorum embryos of the same cocoon are developing very synchronous $[14,24]$. This is especially true for early developmental stages, including germ-disc stage embryos (own observation). As only around $20 \%$ of the Pt-fgf8 RNAi embryos showed a cumulus migration defect phenotype (see Fig. S2) we monitored the development of 68 living embryos (see Fig. S15). Of these 68 embryos $16 \%$ developed the typical tube like Pt-fgf8 knockdown phenotype. We fixed the remaining embryos of this cocoon at late stage 5 to perform the aforementioned experiments. In addition to the pMad antibody staining and to the Pt-dpp in situ hybridisation we analysed 72 embryos for the expression of the cumulus marker PtEts4. This staining revealed that all analysed embryos had developed a regular cumulus. However, in 19 of the 72 embryos (26\%) the cumulus did stay in the centre of the germdisc and these embryos showed a strongly reduced pMad signal (Fig. 3h). The 59 embryos that we analysed for Pt-dpp expression did not reveal a reduced expression for $P t-d p p$ transcripts. Again, in a small portion of the analysed embryos (23\%) the cumulus did not shift towards the rim of the disc and Pt-dpp transcripts were detectable in the centre of the germ-disc (Fig. 3j). Similar to the embryos stained for Pt-Ets4, the pMad signal was strongly reduced in embryos with a centrally located cumulus. Finally, we took the remaining embryos (139 embryos; see Fig. S15) of the described Pt-fgf8 RNAi cocoon and performed a pMad antibody staining. 20 of the 139 embryos (14\%) showed a reduced pMad activity (Fig. S15). Overall, the experiment indicated that FGFR signalling is not influencing the expression of the BMP ligand Pt-dpp but is blocking the activation of the BMP signalling pathway downstream of Dpp. 


\section{Pt-fgf8 expression is regulated via the Hh signalling pathway}

A genome wide search for genes being regulated by the Hh signalling pathway showed that Pt-fgf8 expression levels were significantly altered upon Pt-hh and Pt-ptc RNAi [23]. As our experiments indicate that Pt-fgf8 might be an important factor controlling cumulus migration, we repeated the published Pt-ptc and Pt-hh knockdown experiments and analysed the expression of Pt-fgf8 in stage 5 embryos. Our experiments confirmed the results by Akiyama-Oda and Oda and showed that Pt-fgf8 transcripts are no longer detectable in Pt-hh RNAi embryos (Fig. 3 k, l). In contrast, knockdown of Pt-ptc did lead to an ectopic, central activation of $P t-f g f 8$ expression at stage 5 of embryonic development. As discussed below (see discussion and Fig. 4) these results are in accordance with published cumulus migration defect phenotypes that were observed in Pt-ptc and Pt-hh knockdown embryos and favour a hypothetical model in which Fgf8 acts as a chemo attractant to guide cumulus cells towards the rim of the germ-disc.

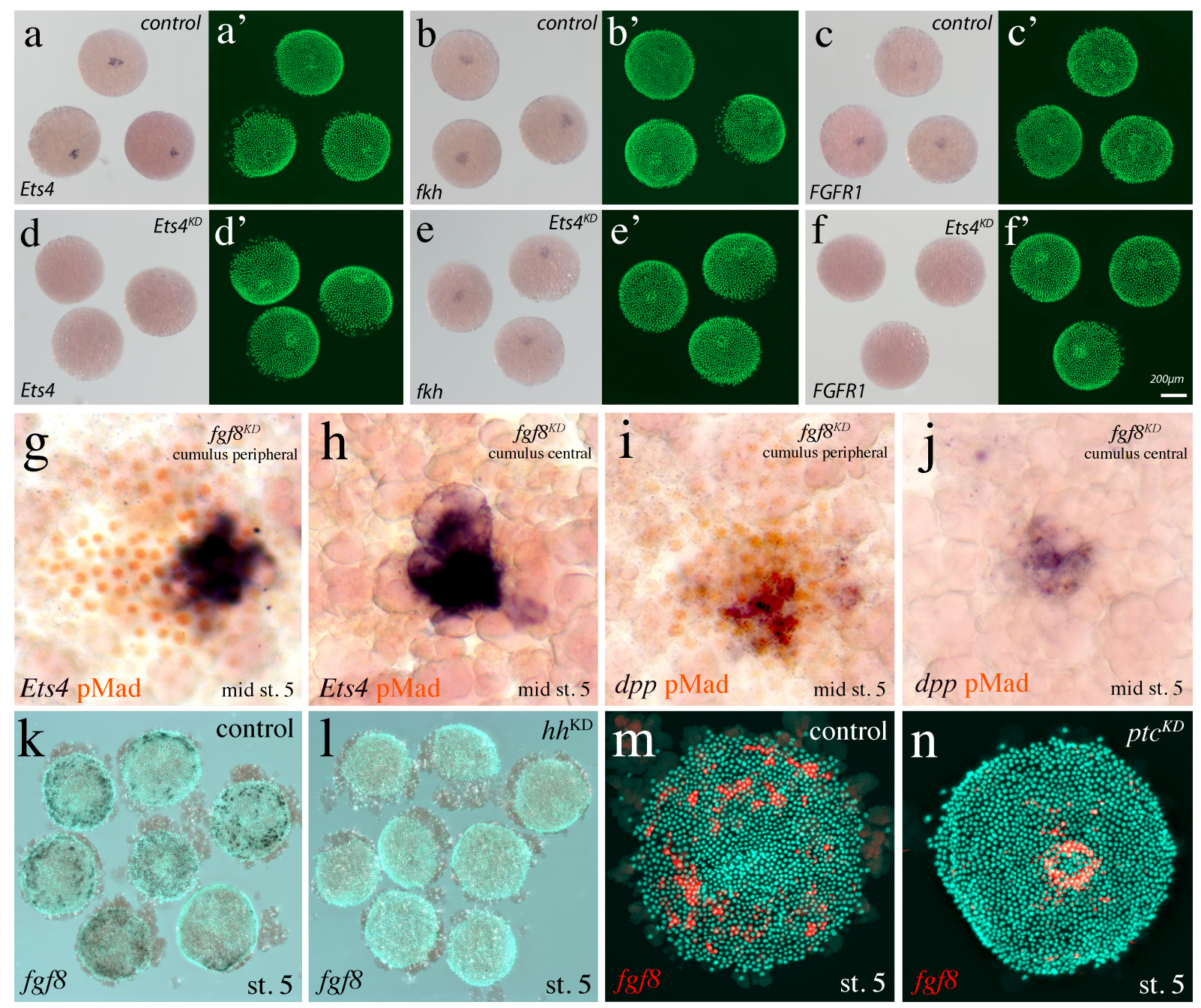

Fig. 3 Upstream and downstream of FGF signalling. (a-f) FGF signalling components are regulated by Pt-Ets4 (also compare to Table1 and Fig. S5). (a, a' and d, d') Pt-Ets4 expression in control and Pt-Ets4 RNAi embryos. (b, b' and e, e') Positive control. As 
shown before (Pechmann et al. 2017), Pt-Ets4 is not influencing the expression of Pt-fkh in the cumulus. (c, $\mathbf{c}^{\prime}$ and $\mathbf{f}, \mathbf{f}^{\prime}$ ) The expression of Pt-FGFR1 is absent in Pt-Ets4 RNAi embryos. (g-j) Knockdown of Pt-fgf8 is influencing the activation of the BMP signalling pathway. BMP signalling pathway activation visualized via pMad antibody staining (orange nuclear staining) in Pt-fgf8 RNAi embryos. Pt-fgf8 knockdown embryos show no obvious reduction in Pt-Ets4 or Pt-dpp expression. In Pt-fgf8 knockdown embryos that lack cumulus migration (h, $\mathbf{j}$ ) also pMad activation is strongly reduced. (k-n) Hedgehog signalling is influencing Pt-fgfo expression in germ-disc embryos. Pt-fgfo expression is absent in Pt-hh RNAi embryos $(\mathbf{k}, \mathbf{l})$. Knockdown of the receptor Pt-ptc is leading to ectopic activation of Pt-fgf8 in the centre of the germ-disc (m, n).

\section{Discussion}

During embryogenesis of most animals, BMP signalling is involved in setting up the dorsoventral (DV) body axis (reviewed in e.g. [42]). In spiders, the migrating cumulus is the source of this DV axis inducing BMP signal. In this arthropod system, the controlled localization of the BMP signal (via local secretion of Dpp from cumulus cells) is necessary and sufficient to induce dorsoventral body axis formation [16,18,19,21]. A failure in cumulus migration, the loss of the cumulus or the loss of the BMP signal, results in severe DV body axis patterning defects (this study, [16,18,19,22]).

Here we show, that FGF signalling is involved in cumulus migration in the spider $P$. tepidariorum. We provide evidence that FGF signalling is regulated by the transcription factor Ets4 and via the Hh signalling pathway. Finally, we show that FGF signalling is also involved in BMP signal transduction and we provide a hypothetical model in which cumulus migration might be triggered by the asymmetric localization of FGFs.

\section{FGF signalling is involved in cumulus migration in spiders}

The observation that the knockdown of the FGFR receptors (this study, [22]) are not leading to any detectable cumulus migration phenotype and that the knockdown of the ligand Pt-fgf8 and the downstream factor Pt-dof is only leading to a phenotype in a small portion of embryos indicate that this signalling pathway is not very sensitive to RNAi, at least in P. tepidariorum. It is very likely that small amounts of FGFR ligand as well as remnants (or maternally provided) FGF receptors are already sufficient to initiate normal cumulus migration and signalling. Nevertheless, the fact that we observed a similar FGF signalling phenotype for the ligand (Pt-fgf8) as well as for the downstream component (Pt-dof) reveals that FGF signalling has a role in cumulus migration in the spider. 
Our expression analysis clearly shows that in evolutionary younger spiders like $P$. tepidariorum as well as in the basally branching spider, like the tarantula A. geniculata, several components of the FGF signalling pathway are expressed either in the germ-disc (Pt-fgf8, Sg-fgf8, Ag-fgf8 and Ag-fgf17) or in the cumulus itself (Pt-dof, Ag-dof, Pt-FGFR1, Ag-FGFR1). This analysis makes it very likely, that FGF signalling is also involved in cumulus migration in basally branching spiders. Expression analysis of FGF components in other chelicerate species (e.g. Opiliones, scorpions, whip spiders, etc.) would be required to show if cumulus migration via FGF signalling is a synapomorphic character of chelicerates.

Our phylogenetic and expression analysis of FGF ligands indicates that there might be variation in the recruitment of different fibroblast growth factors during the embryogenesis of different spider species. The genome of $P$. tepidariorum is one of the best-assembled spider genomes [26]. In addition, multiple transcriptome assemblies are available for this species [40]. For these reasons, it is very likely that $f g f 17$ is indeed missing from the P. tepidariorum genome. As we could find fgf17 in Steatoda grossa (a closely related cobweb spider), this potential loss of fgf17 might be specific to Parasteatoda and not to cobweb spiders in general. However, as we could not detect any expression of $S g$ - $f g f 17$ during germ-disc stages, $S g$ - $f g f 17$ might not have a function that is related to cumulus migration. This is in contrast to the expression of $A g-f g f 17$ in the tarantula that showed a very similar pattern to $A g-f g f 8$. These results indicate that both, Fgf8 and Fgf17, might have a role during cumulus migration in basally branching spiders like A. geniculata but that this function is restricted to Fgf8 in higher spiders. In turn, this might have facilitated the loss of $f g f 17$ in Parasteatoda.

\section{FGF signalling is controlled via Hh signalling and the transcription factor Ets4}

Our results on the regulation of $P t-f g f 8$ expression within the germ-disc are in agreement with the results of a recent publication, which shows the genome-wide identification of the downstream targets of the Hh signalling pathway [23]. Similar to the results of our study, also in the study by Akiyama-Oda and Oda, Pt-fgf8 was no longer detectable in the cells of the germ-disc, when Pt-hh was reduced via parental RNAi. Furthermore, like in our study, Pt-fgf8 was ectopically activated in the central region of the germ-disc upon down regulation of the negative regulator of the $\mathrm{Hh}$ signalling pathway Pt-ptc (Fig. 3k-n). 
Previously, we identified the transcription factor Ets4 as an important player for dorsoventral axis formation in P. tepidariorum [19]. On the one hand, down-regulation of Pt-Ets4 did lead to a loss of the integrity of the cumulus cells. As a consequence the cumulus got lost and DV body axis formation was not initiated. On the other hand, ectopic expression of Pt-Ets4 did lead to ectopic cell delamination and to an independent initiation of cell-migration of single cells. These results indicated that Pt-Ets 4 is an important player within the cumulus but is not sufficient on its own to induce the formation of an ectopic cumulus. It is very likely that the combination of several molecular factors is required to set up a fully functional cumulus. To get a better idea of how Pt-Ets4 is regulating different processes within the cells of the cumulus, we performed an RNA-seq experiment and compared the expression profile of Pt-Ets4 RNAi cumuli with those of cumuli of control embryos. This analysis clearly showed that the early activation of several components of the FGF signalling pathway within the developing cumulus cells seems to be under the control of Pt-Ets4 (Table 1). As FGF signalling is clearly involved in cell migration, this analysis indicates a link between PtEts4 and cumulus cell migration.

\section{A hypothetical model for cumulus cell migration in spiders}

During early spider embryogenesis the cumulus shifts from the centre to the periphery of the radially symmetric germ-disc. It is still unclear if this is a completely stochastic process or if the cumulus is directed towards the rim of the disc via an unknown mechanism. Life imaging of cumulus migration in different spider species indicates that the movement of the cumulus towards the rim of the disc is relatively straight, once the cumulus has started to migrate (see Movie S1 and 2, e.g. [22,24,43,44]). This holds even true for very huge tarantula embryos [18]. It was noted that in around $25 \%$ of wild type P. tepidariorum embryos, the cumulus is not perfectly centred within the germ-disc but is slightly asymmetrically positioned. However, even in these embryos the cumulus movement seems to not correlate with the positioning of the cumulus within the germdisc and cumulus cells do not necessarily take the shortest distance to reach the rim of the disc [13]. This observation indicates that an unknown mechanism might be responsible to give a direction to the shifting cumulus cells.

Although the expression of $P t-f g f 8$ is highly variable in germ-disc stage $P$. tepidariorum embryos, we would like to hypothesise that the stochastic and often very asymmetric expression of $P t-f g f 8$ during stage 4 embryos (before cumulus migration, e.g. Fig. 2 d, e) 
might set up a short-term concentration gradient of Fgf8 protein across the early stage 4 germ-disc (Fig. 4a). This FGF gradient might be interpreted by the cumulus cells and could be responsible for the initial directional movement of the cumulus cells. Depending on the strength of this asymmetric or more uniform Pt-fgf8 expression the hypothetical Fgf8 protein gradient could either be relatively steep or be more flat.

Studies in other animal model systems were able to show that FGFs can act as a chemoattractant (e.g. [7,8], reviewed in [6]). Our observation that $P$. tepidariorum cumulus cells, at late stage 5, always stop their migration at high levels of Pt-fgf8 expression (Fig. 2i, j, k, Fig. S8 and S9) indicates that also in the spider Fgf8 might act as a chemoattractant.

This hypothesis is also supported by the differences in Pt-fgf8 expression that can be found when the expression of different components of the Hh signalling pathway are reduced via RNAi. As indicated in Fig. 4b, these differences in Pt-fgf8 expression and the resulting putative differences in the Fgf8 protein gradients, might explain the observed alterations in cumulus migration (this study, [22,23]). Briefly, in strongly affected Pt-hh as well as in Pt-ptc RNAi embryos cumulus migration was prevented [22]. This loss of cumulus migration could be explained by the loss (in Pt-hh RNAi) or the ectopic central activation (in Pt-ptc RNAi) of Pt-fgf8 expression. In the case of the loss of $P t-f g f 8$ expression in Pt-hh RNAi embryos, cumulus attraction via Pt-fgf8 might be missing. As a result, cumulus migration is not initiated. In contrast, the ectopic central Pt-fgf8 activation in Pt-ptc RNAi embryos might strongly attract and keep the cumulus cells in the middle of the germ-disc (Fig. 4b). Our expression analysis indicates that the clearance of $P t-f g f 8$ transcripts from the centre of the stage 5 germ-disc, is an important aspect of the dynamic Pt-fgf8 expression. This observation further supports the idea that the central ectopic expression of $P t-f g f 8$ in Pt-ptc knockdown embryos prevents the cumulus from shifting.

In P. tepidariorum, Hh signalling is heavily involved in patterning the anteroposterior axis of the germ-disc $[22,23]$. This leads to the suggestion that Hh signalling might set up a positional value gradient across the germ-disc and that the cumulus cells move down along this emerging positional value gradient [22]. In this study it was also noted that Hh protein in vertebrate embryos might be able to travel a distance of around $300 \mu \mathrm{m}$ and that this distance is very similar to the radius of $P$. tepidariorum germ-disc stage embryos [22,45]. However, the size of spider eggs and embryos is very diverse. In $A$. geniculata the radius of the germ-disc is already around $1 \mathrm{~mm}$ [18] and the embryos of 
this species are not the biggest amongst Mygalomorphae. For this reason we propose a different mechanism in which the cumulus on its own is driving the activation of a chemo-attractant in a posterior (centre of the germ-disc) to anterior (rim of the germdisc) direction. This model is based on the observation that we could detect strong Ptfgf8 expression at the anterior border of the bulged germ-disc ectoderm (e.g. Fig. 2 i, several embryos in Fig. S8). The bulging is a consequence of the moving cumulus, which shifts underneath the germ-disc and is mechanically pushing the ectoderm upwards (see Fig. 4a, [22]). In our hypothetical model this cumulus driven bulging induces mechanical stress in the overlaying ectoderm, which in turn could trigger the further activation of fgf8 expression in front of the migrating cumulus. It was shown that mechanically induced stretching or compression of cells is able to induce receptor activation and gene expression, including fibroblast growth factor receptors and ligands (e.g. [46,47]).

As our model is a kind of self-enhancing system and does not depend on a stable and long lasting long-range morphogen gradient, it might be functional in any spider embryo, regardless of the size of the germ-disc.

Alternative mechanisms could also lead to the local enhancement of FGFs within certain regions of the germ-disc, and this might then lead to the local attraction of cumulus cells. In zebrafish lateral line formation, it was shown that apical constriction leads to the formation of microlumen, which are in the centre of rosette-like structures. These structures are responsible to trap secreted FGFs and are able to increase signalling responses and the coordination of migratory cell behaviour (reviewed in [48], original article [49]). Future studies are required to proof if our model is valuable. 


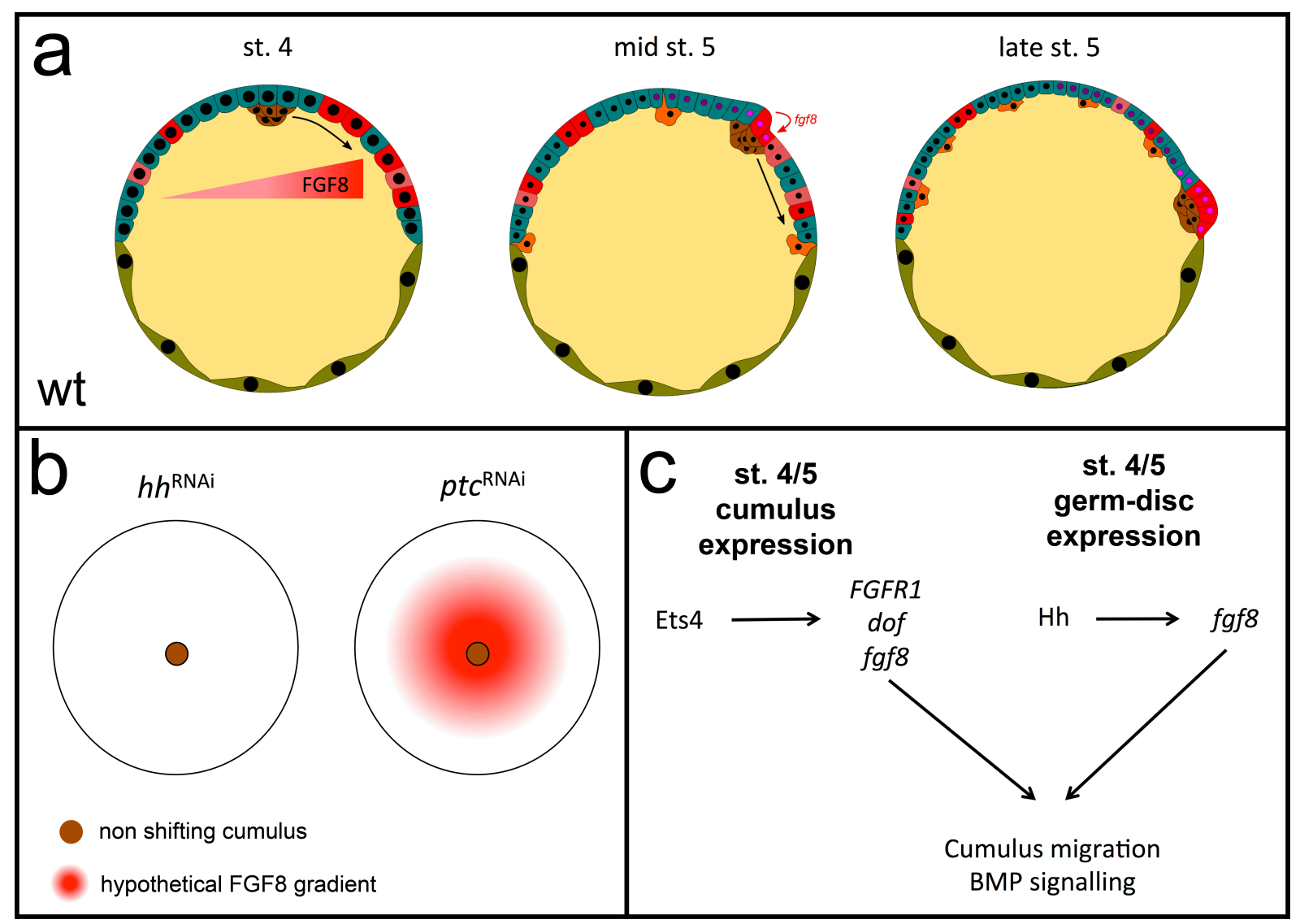

Fig. 4 Hypothetical diagram of cumulus migration and the regulation of the FGF signalling pathway in Parasteatoda. (a) The diagram shows a cross-section of wild type germ-disc stage embryos. At early germ-disc stage (stage 4), stochastic expression of $f g f 8$ might result in a stochastic distribution of Fgf8 protein shortly before cumulus cells start to migrate. Cumulus cells are able to interpret the strength of this Fgf8 gradient (red graded triangle) and start to migrate towards high levels of Fgf8 (black curved arrow at st. 4). Please note: In this hypothetical model a cumulus attracting longrange FGF protein gradient is only required shortly before the cumulus starts to migrate (at st. 4). As soon as cumulus migration is initiated, the bulging of the overlaying ectoderm or another unknown mechanism might enhance $f g f 8$ expression (red curved arrow at mid st. 5) at the anterior border of the migrating cumulus. This process might further attract and could explain the straight movement (black arrow) of the cumulus cells towards the rim of the germ-disc. At the end of stage 5 the cumulus halts its movement at high levels of $f g f 8$ expression. Colour code: germ-disc cells - blue; extraembryonic cells - green; yolk - yellow; fgfo expressing germ-disc cells - light and dark red; gastrulating cells - orange; cumulus cells - brown. Dark and light magenta colouring indicates former and present pMad positive nuclei of the ectoderm (the BMP signalling pathway gets activated by the Dpp secreting cumulus cells). (b) Top view on the germ-disc of stage 5 RNAi embryos. The brown circle indicates the cumulus that is not shifting in $h h$ and ptc RNAi embryos. In the ptc RNAi embryo, hypothetical Fgf8 protein levels are indicated in red shading. In the wild-type, the cumulus is shifting straight towards the rim of the disc and stops at high levels of Fgf8 (compare to a). The cumulus of strongly affected $h h$ and ptc RNAi embryos does not shift because of the missing (in $h h$ RNAi, see Fig. 3l) or the ectopic, central (in ptc RNAi, see Fig. 3n) expression of $f g f 8$. (c) Simplified gene regulatory network (GRN) that is responsible for the regulation of components of the FGF signalling pathway in the cumulus and in the ectoderm of the germ-disc. 


\section{Future directions}

Our results indicate that Pt-fgf8 is also required to activate the BMP signalling pathway in ectodermal germ-disc cells overlaying the cells of the cumulus. In wild-type, this activation is crucial to induce the dorsal field at the rim of the disc. This in turn is required to break the radially symmetry of the germ-disc (e.g. [15,16]).

The performed analysis indicates that the involvement of Pt-Fgf8 in activating the BMP signalling pathway is probably not on the level of ligand (Pt-dpp expression was not affected) activation but might be downstream of receptor activation. Future studies, like a genome wide search of FGFR target genes might help to understand, if FGFs are really required to activate the BMP signalling pathway or if other mechanism are causing the observed phenotype. In Drosophila it was shown that FGF signalling is involved in cytoneme formation (e.g. [50]). Also in P. tepidariorum germ-disc embryos cytoplasmic cytoneme like structures that were projecting from the cumulus towards the germ-disc epithelium could be identified [13]. Cytonemes are known to be important players in the BMP signalling pathway [51-53]. For this reason, missing cytonemes might be the reason that strongly affected $f g f 8$ knockdown embryos are lacking an active BMP signalling pathway.

Unfortunately, we failed to regionally down-regulate (via embryonic RNAi) or overactivate Pt-fgf8 expression (via embryonic capped mRNA injection) in P. tepidariorum. In the future, also cell culture experiments could be useful to analyse if Fgf8 is indeed able to attract cumulus cells.

Recently, BMP soaked agarose beads were successfully transplanted to germ-disc stage tarantula embryos [18]. These BMP beads were able to induce a secondary axis in $A$. geniculata. Similar experiments should also be performed with Fgf8/17 soaked beads. 


\section{Acknowledgements}

We thank Siegfried Roth for commenting on the manuscript.

\section{Disclaimer}

The funders had no role in study design, data collection and analysis, decision to publish or preparation of the manuscript.

\section{Funding}

This work has been funded by the Deutsche Forschungsgemeinschaft (DFG grant PE 2075/1-2 and PE 2075/3-1 to M.P. and DFG grant SCHI 1365/2-1 to P.S.).

\section{Competing interests}

We declare we have no competing interests

\section{Authors' contributions}

M.P. designed the study, performed RNAi experiments and the phylogenetic analysis and wrote the paper. R.W. performed the RNA sequencing experiment, performed parts of the molecular and bioinformatics analysis and commented on the manuscript. P.S. performed the initial bioinformatics analysis of the RNA seq. data and commented on the manuscript. L.K. performed in situ analysis in A. geniculata and commented on the manuscript. All authors gave final approval for publication.

\section{References}

1. Xie Y et al. 2020 FGF/FGFR signaling in health and disease. Signal Transduct Target Ther 5, 181. (doi:10.1038/s41392-020-00222-7)

2. Dorey K, Amaya E. $2010 \mathrm{FGF}$ signalling: diverse roles during early vertebrate embryogenesis. Development 137, 3731-3742. (doi:10.1242/dev.037689)

3. Beermann A, Schröder R. 2008 Sites of Fgf signalling and perception during embryogenesis of the beetle Tribolium castaneum. Dev Genes Evol 218, 153-167. (doi:10.1007/s00427-007-0192-x)

4. Sharma R, Beer K, Iwanov K, Schmöhl F, Beckmann PI, Schröder R. 2015 The single fgf receptor gene in the beetle Tribolium castaneum codes for two isoforms that integrate FGF8- and Branchless-dependent signals. Dev Biol 402, 264-275.

(doi:10.1016/j.ydbio.2015.04.001)

5. Sharma R, Beermann A, Schröder R. 2013 FGF signalling controls anterior extraembryonic and embryonic fate in the beetle Tribolium. Dev Biol 381, 121-133. (doi:10.1016/j.ydbio.2013.05.031)

6. Muha V, Müller H-AJ. 2013 Functions and Mechanisms of Fibroblast Growth Factor (FGF) Signalling in Drosophila melanogaster. Int J Mol Sci 14, 5920-5937. 
(doi:10.3390/ijms14035920)

7. Sato M, Kornberg TB. 2002 FGF Is an Essential Mitogen and Chemoattractant for the Air Sacs of the Drosophila Tracheal System. Developmental Cell 3, 195-207. (doi:10.1016/S1534-5807(02)00202-2)

8. Clark IBN, Muha V, Klingseisen A, Leptin M, Müller H-AJ. 2011 Fibroblast growth factor signalling controls successive cell behaviours during mesoderm layer formation in Drosophila. Development 138, 2705-2715. (doi:10.1242/dev.060277)

9. Burdine RD, Branda CS, Stern MJ. 1998 EGL-17(FGF) expression coordinates the attraction of the migrating sex myoblasts with vulval induction in C. elegans.

Development 125, 1083-1093.

10. Borland CZ, Schutzman JL, Stern MJ. 2001 Fibroblast growth factor signaling in Caenorhabditis elegans. Bioessays 23, 1120-1130. (doi:10.1002/bies.10007)

11. Lo T-W, Bennett DC, Goodman SJ, Stern MJ. 2010 Caenorhabditis elegans Fibroblast Growth Factor Receptor Signaling Can Occur Independently of the MultiSubstrate Adaptor FRS2. Genetics 185, 537-547. (doi:10.1534/genetics.109.113373) 12. Sun X, Meyers EN, Lewandoski M, Martin GR. 1999 Targeted disruption of Fgf8 causes failure of cell migration in the gastrulating mouse embryo. Genes Dev 13, 18341846. (doi:10.1101/gad.13.14.1834)

13. Akiyama-Oda Y, Oda H. 2003 Early patterning of the spider embryo: a cluster of mesenchymal cells at the cumulus produces Dpp signals received by germ disc epithelial cells. Development 130, 1735-1747. (doi:10.1242/dev.00390)

14. Hilbrant M, Damen WGM, McGregor AP. 2012 Evolutionary crossroads in developmental biology: the spider Parasteatoda tepidariorum. Development 139, 26552662. (doi:10.1242/dev.078204)

15. Oda H, Akiyama-Oda Y. 2008 Differing strategies for forming the arthropod body plan: lessons from Dpp, Sog and Delta in the fly Drosophila and spider Achaearanea. Dev. Growth Differ. 50, 203-214. (doi:10.1111/j.1440-169X.2008.00998.x)

16. Akiyama-Oda Y, Oda H. 2006 Axis specification in the spider embryo: dpp is required for radial-to-axial symmetry transformation and sog for ventral patterning. Development 133, 2347-2357. (doi:10.1242/dev.02400)

17. Schwager EE, Schönauer A, Leite DJ, Sharma PP, McGregor AP. 2015 Chelicerata. In Evolutionary Developmental Biology of Invertebrates 3 (ed A Wanninger), pp. 99-139. Springer Vienna. (doi:10.1007/978-3-7091-1865-8_5)

18. Pechmann M. 2020 Embryonic development and secondary axis induction in the Brazilian white knee tarantula Acanthoscurria geniculata, C. L. Koch, 1841 (Araneae; Mygalomorphae; Theraphosidae). Dev Genes Evol 230, 75-94. (doi:10.1007/s00427020-00653-w)

19. Pechmann M, Benton MA, Kenny NJ, Posnien N, Roth S. 2017 A novel role for Ets4 in axis specification and cell migration in the spider Parasteatoda tepidariorum. Elife 6. (doi:10.7554/eLife.27590)

20. Holm Å. 1952 Experimentelle Untersuchungen über die Entwicklung und Entwicklungsphysiologie des Spinnenembryos. Uppsala: Almqvist \& Wiksells.

21. Oda H, Iwasaki-Yokozawa S, Usui T, Akiyama-Oda Y. 2020 Experimental duplication of bilaterian body axes in spider embryos: Holm's organizer and selfregulation of embryonic fields. Dev Genes Evol 230, 49-63. (doi:10.1007/s00427-01900631-x)

22. Akiyama-Oda Y, Oda H. 2010 Cell migration that orients the dorsoventral axis is coordinated with anteroposterior patterning mediated by Hedgehog signaling in the early spider embryo. Development 137, 1263-1273. (doi:10.1242/dev.045625)

23. Akiyama-Oda Y, Oda H. 2020 Hedgehog signaling controls segmentation 
dynamics and diversity via msx1 in a spider embryo. Science Advances 6, eaba7261. (doi:10.1126/sciadv.aba7261)

24. Mittmann B, Wolff C. 2012 Embryonic development and staging of the cobweb spider Parasteatoda tepidariorum C. L. Koch, 1841 (syn.: Achaearanea tepidariorum; Araneomorphae; Theridiidae). Dev. Genes Evol. 222, 189-216. (doi:10.1007/s00427012-0401-0)

25. Altschul SF, Gish W, Miller W, Myers EW, Lipman DJ. 1990 Basic local alignment search tool. J. Mol. Biol. 215, 403-410. (doi:10.1016/S0022-2836(05)80360-2)

26. Schwager EE et al. 2017 The house spider genome reveals an ancient wholegenome duplication during arachnid evolution. BMC Biol. 15, 62. (doi:10.1186/s12915017-0399-x)

27. Thurmond J et al. 2019 FlyBase 2.0: the next generation. Nucleic Acids Res. 47, D759-D765. (doi:10.1093/nar/gky1003)

28. McGregor AP, Pechmann M, Schwager EE, Feitosa NM, Kruck S, Aranda M, Damen WGM. 2008 Wnt8 Is Required for Growth-Zone Establishment and Development of Opisthosomal Segments in a Spider. Current Biology 18, 1619-1623.

(doi:10.1016/j.cub.2008.08.045)

29. Pechmann M, McGregor AP, Schwager EE, Feitosa NM, Damen WGM. 2009 Dynamic gene expression is required for anterior regionalization in a spider. Proc. Natl. Acad. Sci. U.S.A. 106, 1468-1472. (doi:10.1073/pnas.0811150106)

30. Chen S, Zhou Y, Chen Y, Gu J. 2018 fastp: an ultra-fast all-in-one FASTQ preprocessor. Bioinformatics 34, i884-i890. (doi:10.1093/bioinformatics/bty560)

31. Bray NL, Pimentel H, Melsted P, Pachter L. 2016 Near-optimal probabilistic RNAseq quantification. Nat Biotechnol 34, 525-527. (doi:10.1038/nbt.3519)

32. Edgar RC. 2004 MUSCLE: multiple sequence alignment with high accuracy and high throughput. Nucleic Acids Res 32, 1792-1797. (doi:10.1093/nar/gkh340)

33. Capella-Gutiérrez S, Silla-Martínez JM, Gabaldón T. 2009 trimAl: a tool for automated alignment trimming in large-scale phylogenetic analyses. Bioinformatics 25, 1972-1973. (doi:10.1093/bioinformatics/btp348)

34. Dereeper A et al. 2008 Phylogeny.fr: robust phylogenetic analysis for the nonspecialist. Nucleic Acids Res. 36, W465-469. (doi:10.1093/nar/gkn180)

35. Whelan S, Goldman N. 2001 A general empirical model of protein evolution derived from multiple protein families using a maximum-likelihood approach. Mol. Biol. Evol. 18, 691-699.

36. Prpic N-M, Schoppmeier M, Damen WGM. 2008 Whole-Mount In Situ Hybridization of Spider Embryos. Cold Spring Harb Protoc 2008, pdb.prot5068. (doi:10.1101/pdb.prot5068)

37. Benton MA, Pechmann M, Frey N, Stappert D, Conrads KH, Chen Y-T, Stamataki E, Pavlopoulos A, Roth S. 2016 Toll Genes Have an Ancestral Role in Axis Elongation. Curr. Biol. 26, 1609-1615. (doi:10.1016/j.cub.2016.04.055)

38. Schindelin J et al. 2012 Fiji: an open-source platform for biological-image analysis. Nat. Methods 9, 676-682. (doi:10.1038/nmeth.2019)

39. Popovici C, Roubin R, Coulier F, Birnbaum D. 2005 An evolutionary history of the FGF superfamily. Bioessays 27, 849-857. (doi:10.1002/bies.20261)

40. Posnien $\mathrm{N}$ et al. 2014 A comprehensive reference transcriptome resource for the common house spider Parasteatoda tepidariorum. PLOS ONE 9, e104885.

(doi:10.1371/journal.pone.0104885)

41. Pechmann M. 2016 Formation of the germ-disc in spider embryos by a condensation-like mechanism. Front. Zool. 13, 35. (doi:10.1186/s12983-016-0166-9)

42. De Robertis EM. 2008 Evo-Devo: Variations on Ancestral Themes. Cell 132, 185- 
195. (doi:10.1016/j.cell.2008.01.003)

43. Chaw RC, Vance E, Black SD. 2007 Gastrulation in the spider Zygiella x-notata involves three distinct phases of cell internalization. Dev. Dyn. 236, 3484-3495. (doi:10.1002/dvdy.21371)

44. Wolff C, Hilbrant M. 2011 The embryonic development of the central American wandering spider Cupiennius salei. Front. Zool. 8, 15. (doi:10.1186/1742-9994-8-15)

45. Lewis PM, Dunn MP, McMahon JA, Logan M, Martin JF, St-Jacques B, McMahon AP. 2001 Cholesterol modification of sonic hedgehog is required for long-range signaling activity and effective modulation of signaling by Ptc1. Cell 105, 599-612. (doi:10.1016/s0092-8674(01)00369-5)

46. Li C-F, Hughes-Fulford M. 2006 Fibroblast Growth Factor-2 Is an ImmediateEarly Gene Induced by Mechanical Stress in Osteogenic Cells. Journal of Bone and Mineral Research 21, 946-955. (doi:10.1359/jbmr.060309)

47. Kinoshita N, Hashimoto Y, Yasue N, Suzuki M, Cristea IM, Ueno N. 2020 Mechanical Stress Regulates Epithelial Tissue Integrity and Stiffness through the FGFR/Erk2 Signaling Pathway during Embryogenesis. Cell Reports 30, 3875-3888.e3. (doi:10.1016/j.celrep.2020.02.074)

48. Chan CJ, Heisenberg C-P, Hiiragi T. 2017 Coordination of Morphogenesis and CellFate Specification in Development. Current Biology 27, R1024-R1035.

(doi:10.1016/j.cub.2017.07.010)

49. Durdu S, Iskar M, Revenu C, Schieber N, Kunze A, Bork P, Schwab Y, Gilmour D. 2014 Luminal signalling links cell communication to tissue architecture during organogenesis. Nature 515, 120-124. (doi:10.1038/nature13852)

50. Du L, Sohr A, Yan G, Roy S. 2018 Feedback regulation of cytoneme-mediated transport shapes a tissue-specific FGF morphogen gradient. eLife 7, e38137. (doi:10.7554/eLife.38137)

51. Kornberg TB. 2017 Distributing signaling proteins in space and time: the province of cytonemes. Curr Opin Genet Dev 45, 22-27. (doi:10.1016/j.gde.2017.02.010) 52. Kornberg TB, Roy S. 2014 Cytonemes as specialized signaling filopodia. Development 141, 729-736. (doi:10.1242/dev.086223)

53. Zhang C, Scholpp S. 2019 Cytonemes in development. Current Opinion in Genetics \& Development 57, 25-30. (doi:10.1016/j.gde.2019.06.005) 